\title{
Cosmological CMBR dipole in open universes?
}

\author{
David Langlois \\ Département d'Astrophysique Relativiste et de Cosmologie, \\ UPR 176, Centre National de la Recherche Scientifique, \\ Observatoire de Paris, 92195 Meudon, France
}

April 15, 2018

\begin{abstract}
The observed CMBR dipole is generally interpreted as a Doppler effect arising from the motion of the Earth relative to the CMBR frame. An alternative interpretation, proposed in the last years, is that the dipole results from ultra-large scale isocurvature perturbations. We examine this idea in the context of open cosmologies and show that the isocurvature interpretation is not valid in an open universe, unless it is extremely close to a flat universe, $\left|\Omega_{0}-1\right|<10^{-4}$.
\end{abstract}

\section{Introduction}

In the standard Big Bang scenario, the oldest relic from the early universe is the Cosmic Microwave Background Radiation (CMBR). This radiation is remarkably close to isotropy. When it is decomposed into multipoles,

$$
\frac{\Delta T}{T}(\theta, \phi)=\sum_{l=1}^{\infty} \sum_{m=-l}^{l} a_{l m} Y_{l m}(\theta, \phi)
$$


one finds that the dipole contribution is of the order $(\Delta T / T)_{l=1} \sim 10^{-3}$, while the contribution from higher multipoles is only $(\Delta T / T)_{l>1} \sim 10^{-5}$ (see e.g. [1]). Usually, the dipole term is interpreted as a Doppler effect, i.e. as the consequence of our local motion with respect to the "CMBR rest frame", whereas the other multipoles are accounted for by primordial cosmological perturbations. However, one cannot reject a priori the possibility that a significant part of the dipole originates as well from cosmological perturbations. In fact, this alternative idea was stimulated by the results of Lauer and Postman [2] in 1991, who found a dipole in distant Abell clusters inconsistent with the CMBR dipole. Other observations based on nearby galaxies, IRAS galaxies or distant supernovae tend on the other hand to favour the orthodox interpretation, but are still inconclusive.

Let us be more precise on the distinction between a cosmological dipole and a local dipole. If our universe was perfectly homogeneous and isotropic then there would be no cosmological dipole; a comoving observer would not detect any dipole whereas a non comoving observer would measure a dipole, in this case a purely local dipole, due to his motion. In a perturbed model, it is more delicate to distinguish between a local and a cosmological dipole. The perturbation of any quantity can be decomposed into contributions from different scales. The cosmological dipole is then due to very large scale perturbations, typically scales of the order of the Hubble radius, and the local dipole is due to the small scale component (the dominant one) of the observer peculiar velocity.

The purpose of this paper is to explore the origin of the dipole from a theoretical point of view and to ask whether it is possible, in the context of Friedman-Lemaitre-Robertson-Walker (FLRW) cosmologies with gaussian random fields of linear perturbations, to obtain a cosmological dipole and higher multipoles compatible with the observations. To discriminate between a local Doppler dipole and a cosmological dipole is more, in our view, than a mere academic exercise because the Doppler assumption enters in the quadrupole analysis of the observations. The reason is that our motion would not only induce a dipole but also a quadrupole, and other multipoles.

It was shown by Paczynski and Piran [3] that a strong dipole can be found within a particular Tolman-Bondi model with an inhomogeneous radiative component. In fact, as was shown recently by Langlois and Piran [4], this result can be obtained, more generally, in a perturbed FLRW flat cosmology, from ultra large scale (larger than the current Hubble radius) isocurvature 
perturbations. Here, we reinvestigate this possibility in the context of open cosmologies.

The influence of stochastic cosmological perturbations with wavelengths larger than the current Hubble radius on the CMBR temperature fluctuations was examined initially by Grishchuk and Zel'dovich [5]. Their analysis was restricted to perturbations in a flat background for which the Fourier expansion is available. When one considers an open universe one needs the complicated formalism of mode functions in an hyperbolic space. Recently, Lyth and Woszczyna [6] stressed the importance of supercurvature modes, which had been ignored previously, when dealing with random fields. With this new element, the influence of ultra large scale perturbations on the CMBR for open universes was then investigated in references [6] and [7]. The present work extends these papers in two respects. First, we focus our attention on the dipole, which was not treated previously, and, second, we allow not only for adiabatic perturbations but also for isocurvature perturbations.

The paper is organized as follows. In section 2, we recall the main steps of the derivation of the Sachs-Wolfe effect, responsible of the large angle anisotropies in the CMBR. Our approach follows the work of Panek [8], although our notation is different. The final result is valid for flat or curved FLRW background geometries and is expressed in terms of gauge-invariant variables. Section 3 gives the CMBR multipoles in a flat geometry. Although these results were already given in [4], the actual presentation is original and provides a bridge to the formalism needed for an open cosmology. In section 4, the case of an open cosmology is considered. Our notation follows essentially Refs [6] and [7]. Finally, section 5 contains the conclusions of this work.

\section{The Sachs-Wolfe effect}

Our background geometry is described by a FLRW metric,

$$
d s^{2}=a^{2}(\eta)\left(-d \eta^{2}+\gamma_{i j} d x^{i} d x^{j}\right)
$$

where $d l^{2}=\gamma_{i j} d x^{i} d x^{j}$ is the metric of a flat or curved maximally symmetric space (a flat space corresponds to $K=0$ and an open space to $K=-1$, where $K$ is the normalized space curvature). The CMBR is composed of cosmological photons, which became free after the recombination of protons 
and electrons and the subsequent decoupling of matter and radiation at a redshift $z_{l s}$. For numerical applications we shall take $z_{l s}=1000\left(z_{l s}\right.$ depends in fact on the spatial curvature but this dependence is very weak 9] and can be safely ignored here). All the photons reaching us now were emitted on a physical surface at the epoch of decoupling, called the last scattering surface, and defined physically by $n_{e}=$ const, where $n_{e}$ is the density of free electrons. The universe will be supposed to be matter dominated from the last scattering time to now, which is an excellent approximation except for low values of $\Omega_{0}$.

Now, we consider a perturbed FLRW universe, endowed with the metric (in the longitudinal gauge)

$$
d s^{2}=a^{2}(\eta)\left[-(1+2 \Phi) d \eta^{2}+(1-2 \Psi) \gamma_{i j} d x^{i} d x^{j}\right] .
$$

Only perturbations of the scalar type will be considered here (see e.g. [10]) and, for simplicity, the anisotropic stress of the matter will be supposed to vanish so that the Einstein equations yield

$$
\Phi=\Psi
$$

Because of the presence of geometric perturbations, photons will be redshifted with slight differences depending on their position of emission on the last scattering surface. The resulting fluctuations in the temperature measured by an observer were first calculated by Sachs and Wolfe [11. The origin of this effect is purely geometric and is dominant only for large angular scales, which correspond essentially to scales larger than the Hubble radius at the epoch of last scattering. On smaller scales, causal processes must be taken into account. In this work, we are interested only by the first multipoles of the CMBR, which are dominated by the last scattering superhorizon modes, so that only the Sachs-Wolfe effect is relevant. In the following, the Sachs-Wolfe effect is rederived shortly following the more modern calculation of Panek [8]. However our notations are different and the final result is expressed in another form.

Denoting $k^{\mu}$ the vector tangent to a null geodesic, the evolution of the photon is governed by the geodesic equation:

$$
k^{\nu} \partial_{\nu} k^{\mu}+\Gamma_{\sigma \tau}^{\mu} k^{\sigma} k^{\tau}=0
$$


where the $\Gamma_{\sigma \tau}^{\mu}$ are the Christoffel symbols. We write the perturbed tangent vector $k^{\mu}=\left\{\nu(1-M),-\nu e^{i}+P^{i}\right\}$, where the terms in $\nu$ represent the unperturbed solution; $e^{i}$ is a unit spatial vector, i.e. such that $\gamma_{i j} e^{i} e^{j}=1$; $M$ is the perturbation of the frequency, $P^{i}$ the perturbation of the spatial direction. Here, we are interested only in the frequency perturbation and thus keep only the time component of the geodesic equation. After using the identity $g_{\mu \nu} k^{\mu} k^{\nu}=0$ at first order, which reads

$$
\gamma_{i j} e^{i} P^{j}=(M-\Phi-\Psi) \nu
$$

in order to eliminate the $P^{i}$ in the time component of the geodesic equation at first order, one finds eventually

$$
\frac{d M}{d \lambda} \equiv\left(\partial_{\eta}-e^{i} \partial_{i}\right) M=\Phi^{\prime}-2 e^{i} \partial_{i} \Phi-\Psi^{\prime}
$$

The prime stands for a derivative with respect to the conformal time $\eta$. Note that the parameter $\lambda$ is related to the affine parameter $\tau$ of the unperturbed geodesic by the relation $d \tau / d \lambda=1 / \nu$. The prime stands for derivative with respect to the conformal time $\eta$. Equation (17) corresponds to Eq. (29) of [8].

Typically one can model the content of the universe from the epoch of the last scattering to now by two fluids, a pressureless baryon component and a radiation component, described locally by a black-body spectrum. Let us define the temperature of the photons as seen by observers moving with the baryon component, so that the temperature ratio between emission and reception is given by

$$
\frac{T_{R}}{T_{E}}=\frac{\left(k^{\mu} u_{\mu}^{b}\right)_{R}}{\left(k^{\mu} u_{\mu}^{b}\right)_{E}}
$$

In the perturbed geometry (3), one thus obtains

$$
\frac{T_{R}}{T_{E}}=\frac{a_{E}}{a_{R}}\left\{1+\left[\Phi-M+e^{i} \partial_{i} V\right]_{E}^{R}\right\},
$$

where the velocity potential $V$ is defined by $\delta u_{i}^{b} \equiv a \partial_{i} V$. As a consequence, the temperature fluctuations on the sky measured by an observer today (denoted by the subscript 0) are given by

$$
\left(\frac{\delta T}{T}\right)_{0}(\theta, \phi)=\left(\frac{\delta T}{T}\right)_{e}+\left(\frac{\delta a}{a}\right)_{e}+\left[\Phi-M+e^{i} \partial_{i} V\right]_{e}^{0} .
$$


The left hand side term is a function of the celestial coordinates corresponding to the direction of observation for the observer. The right hand side is expressed as a function of the emission point, of coordinates $\left(\eta_{e}, x_{e}^{i}\right)$, defined as the intersection of the last scattering surface with the null geodesic going through the observer with the given direction, as well as the observer position $\left(\eta_{0}, x_{0}^{i}\right)$. In general the physical last scattering surface is distinct from the constant time hypersurface $\eta=\eta_{l s}$, but one can replace the first two terms on the right hand side of (10) by $(\delta T / T)\left(\eta_{l s}, x_{l s}^{i} \simeq x_{e}^{i}\right)$ (i.e. as a function of the intersection point between the light geodesic and the hypersurface $\left.\eta=\eta_{l s}\right)$. This follows from the local conservation law $a T=$ const for free radiation. Then, expressing the term $[\Phi-M]_{e}^{0}$ in (10) as an integral over the null geodesic and using equation (7), one finds

$$
\left(\frac{\delta T}{T}\right)_{R}=\frac{1}{4} \delta_{\gamma \mid l s}+(h V)_{l s}+\left[-\Phi+e^{i} \partial_{i} V\right]_{l s}^{0}+\int_{l s}^{0} d \lambda\left(\Phi^{\prime}+\Psi^{\prime}\right),
$$

where $h \equiv a^{\prime} / a$. Using the Stefan law $\rho_{\gamma} \propto T^{4}$, we have replaced the temperature fluctuations by fluctuations of the radiation energy density and also introduced the comoving energy density perturbation $\delta$, which can be derived from the energy density perturbation in the longitudinal gauge by the expression $\delta=(\delta \rho / \rho)_{L}+\left(\rho^{\prime} / \rho\right) V$. To obtain (11), we have used implicitly the equality of the velocities of radiation and baryonic matter at the time of last scattering, which results from the preexisting tight coupling between the two fluids. The term $\delta_{\gamma} / 4$ in (11) represents the intrinsic temperature fluctuations on the last scattering surface. The rest of the expression is the Sachs-Wolfe effect. In (11) all the quantities at the last scattering epoch are now evaluated on the hypersurface $\eta_{l s}$ which does not necessarily coincides with the physical last scattering surface (but all the corrections would be second order perturbations).

It is instructive to decompose the Sachs-Wolfe expression into several components and to rewrite (11), after dropping the term $-\Phi_{0}$ which contributes only to the monopole, in the form

$$
\left(\frac{\delta T}{T}\right)_{R}=\left(\frac{\delta T}{T}\right)_{i n t}+\left(\frac{\delta T}{T}\right)_{p S W}+\left(\frac{\delta T}{T}\right)_{D o p}+\left(\frac{\delta T}{T}\right)_{I S W},
$$


with

$$
\left(\frac{\delta T}{T}\right)_{i n t}=\frac{1}{4} \delta_{\gamma \mid l s}, \quad\left(\frac{\delta T}{T}\right)_{p S W}=(\Phi+h V)_{l s}, \quad\left(\frac{\delta T}{T}\right)_{D o p}=e^{i} \partial_{i}\left(V_{0}-V_{l s}\right),
$$

and

$$
\left(\frac{\delta T}{T}\right)_{I S W}=\int_{l s}^{0} d \lambda\left(\Phi^{\prime}+\Psi^{\prime}\right) .
$$

The term $(\Phi+h V)_{l s}$ will be called the proper (or ordinary) Sachs-Wolfe effect (pSW), because only this term was computed in the pioneering paper of Sachs and Wolfe [1]. The term $e^{i} \partial_{i}\left(V_{0}-V_{l s}\right)$ is the difference between the observer velocity and the emission velocity along the line of sight and corresponds to a Doppler effect (Dop). Note that the local dipole is due only to a term of the form $e^{i} v_{i}^{0}$, where $v_{i}^{0}$ is the contribution of small scales to our peculiar velocity; this contribution, which comes from the nonlinear evolution of the perturbations, will be ignored in the rest of the paper where we consider only the effect of very large scales. Finally the integral term is usually called the integrated Sachs-Wolfe effect (ISW).

The linearized Einstein equations and fluid equations (see e.g. [12] or [13]) can then be used to express all the terms in (11) in terms of only one quantity, the most convenient being the gravitational potential $\Phi$. For example, the velocity potential $V$ can be related to $\Phi$ via the Euler equation and the conservation equation:

$$
V=-\frac{2}{3\left(h^{2}+K\right)}\left(\Phi^{\prime}+h \Phi\right) .
$$

Via the Einstein equations, one gets a relativistic generalization of Poisson equation relating the gravitational potential to the total comoving energy density $\delta_{T}$, which reads:

$$
(\triangle+3 K) \Phi=\frac{3}{2}\left(h^{2}+K\right) \delta_{T}
$$

At this stage one must distinguish two kinds of perturbations: adiabatic and isocurvature perturbations. Any general perturbation can be seen as a linear combination of these two kinds. An isocurvature perturbation corresponds to a primordial (in the radiation era) perturbation of the matter which does 
not affect the geometry: it implies a perturbation in the relative composition of the cosmological fluid with no perturbation in the total energy density. On the contrary an adiabatic perturbation is a primordial perturbation in the total energy density without modification of the relative quantities of the fluids. For an adiabatic perturbation, the relation between the matter and radiation density perturbations is by definition $\delta_{\gamma}=(4 / 3) \delta_{m}$, which implies, during matter domination,

$$
\delta_{\gamma} \simeq \frac{4}{3} \delta_{T}
$$

As will be seen later, the consequence of this relation together with the Poisson equation (16) is that, both for the flat and open cases, the intrinsic fluctuations resulting from adiabatic initial perturbations on scales larger than the Hubble radius are always negligible. For an isocurvature perturbation, during matter domination,

$$
\delta_{\gamma} \simeq-\frac{4}{3} S
$$

where $S=\delta_{m}-(3 / 4) \delta_{\gamma}$ is the entropy perturbation. Both for open and flat universes, the appropriate combination of the equations of motion (i.e. conservation and Euler equations) of the two fluids, radiation and baryons, tells us that $S$ is constant on scales bigger than the Hubble radius, which is the case for perturbations we are interested in. Isocurvature perturbations also produce a gravitational potential. Using the conservation and Euler equations for the two fluids, one obtains that the gravitational potential is given, at the beginning of the matter era, by

$$
\Phi=-\frac{1}{5} S
$$

for the modes larger than the Hubble radius.

To summarize, the multipole coefficients of (1) can be decomposed into the sum of contributions

$$
a_{l m}=a_{l m}^{i n t}+a_{l m}^{S W}=a_{l m}^{i n t}+a_{l m}^{p S W}+a_{l m}^{D o p}+a_{l m}^{I S W} .
$$

The rest of the paper will consist in evaluating the various terms of this expression for open/flat universes with adiabatic/isocurvature perturbations. 


\section{Flat universe}

In this section, we consider the flat FLRW models. To study the CMBR fluctuations, it is more convenient to work in spherical coordinates with the observer at the center, naturally adapted to observations on the celestial sphere, rather than the more usual cartesian coordinates:

$$
d l^{2}=d r^{2}+r^{2}\left(d \theta^{2}+\sin ^{2} \theta d \phi^{2}\right) .
$$

Any perturbation $f(r, \theta, \phi)$ can then be decomposed into

$$
f(r, \theta, \phi, \eta)=\int d k \sum_{l m} f_{k l m}(\eta) Q_{k l m}
$$

where the mode functions $Q_{k l m}$ are the eigenfunctions of the spatial Laplacian with the eigenvalues $-k^{2}$ and are defined by

$$
Q_{k l m}=\sqrt{\frac{2}{\pi}} k j_{l}(k r) Y_{l m}(\theta, \phi),
$$

where the $j_{l}$ are the spherical Bessel functions and $Y_{l m}$ are the spherical harmonics. The definition of the $Q_{k l m}$ is such that they are normalized i.e.

$$
\int r^{2} d r \sin \theta d \theta d \phi Q_{k l m}^{*} Q_{k^{\prime} l^{\prime} m^{\prime}}=\delta\left(k-k^{\prime}\right) \delta_{l l^{\prime}} \delta_{m m^{\prime}}
$$

This decomposition is the equivalent of the Fourier decomposition but in a system of spherical coordinates. This presentation is a good introduction to the open case where the spherical coordinates are the most natural spatial cooordinates.

All the quantities introduced in the previous section can now be decomposed on the mode functions $Q_{k l m}$. As announced before, we shall focus essentially on the gravitational potential $\Phi$. The linearized Einstein equations yield a second order differential equation for the evolution of each mode $\Phi_{k l m}$, which shows that the modes larger than the Hubble radius, i.e. $k<h$, are constant in time in a flat universe during the matter era. This result has several consequences. The first is that the ISW contribution vanishes. The second is that the relation (15) simplifies to

$$
h V=-\frac{2}{3} \Phi, \quad(K=0)
$$


Now, using the orthonormality property (24) with equations (11) and (25), the Sachs-Wolfe harmonic coefficients can be expressed in the form

$$
a_{l m}^{S W}=\sqrt{\frac{2}{\pi}} \int_{0}^{\infty} d k k \frac{\Phi_{k l m}}{3} \mathcal{W}_{l}^{S W}(k),
$$

with the window function $\mathcal{W}_{l}^{S W}(k)=\mathcal{W}_{l}^{p S W}(k)+\mathcal{W}_{l}^{D o p}(k)$, the proper SW and Doppler contributions being respectively given by

$$
\mathcal{W}_{l}^{p S W}(k)=j_{l}\left(k r_{l s}\right), \quad \mathcal{W}_{l}^{D o p}(k)=2 h_{l s}^{-1} \frac{d}{d r} j_{l}\left(k r_{l s}\right)-2 h_{0}^{-1} \frac{d}{d r} j_{l}\left(k r_{0}\right) .
$$

$r_{l s}$ is the comoving distance from the hypersurface $\eta=\eta_{l s}$. It is related to the redshift $z_{l s}\left(\equiv\left(a_{0} / a_{l s}\right)-1\right)$ by the relation

$$
r_{l s}=2 h_{0}^{-1}\left(1-\left(1+z_{l s}\right)^{-1 / 2}\right)=2\left(h_{0}^{-1}-h_{l s}^{-1}\right) .
$$

Using the relations

$$
\frac{d}{d r} j_{l}(k r)=k j_{l-1}(k r)-\frac{l+1}{r} j_{l}(k r), \quad(2 l+1) j_{l}(x)=x\left(j_{l+1}+j_{l-1}(x)\right),
$$

one can rewrite the dipole term

$$
\mathcal{W}_{1}^{S W}(k)=\frac{2}{3} k h_{0}^{-1}\left(j_{0}\left(k r_{l s}\right)-1\right)+\frac{k r_{l s}}{3}\left(1-4 h_{l s}^{-1} r_{l s}^{-1}\right) j_{2}\left(k r_{l s}\right)
$$

and the terms $l>1$

$$
\mathcal{W}_{l}^{S W}(k)=\left(1+\frac{2 l}{h_{l s} r_{l s}}\right) j_{l}\left(k r_{l s}\right)-\frac{2 k}{h_{l s}} j_{l+1}\left(k r_{l s}\right) .
$$

Since $j_{0}(x)=\sin x / x$ and $j_{l}(x) \sim x^{l}$ for $x<<1$, the dipole term $\mathcal{W}_{1}^{S W}(k)$ behaves like $\left(k r_{l s}\right)^{3}$ for small $k$. On the other hand, for $l>1, \mathcal{W}_{l}^{S W}(k)$ goes like $\left(k r_{l s}\right)^{l}$ for long wavelengths. This means that the Sachs-Wolfe dipole on ultra large scales is suppressed with respect to the quadrupole. Let us now consider the intrinsic contribution. In the case of adiabatic perturbations, equation (17) and the Poisson equation (16) (with $K=0$ ) shows that the intrinsic contribution is always negligible with respect to the Sachs-Wolfe contribution for modes larger than the Hubble radius at the time of last scattering. For 
adiabatic perturbations, the temperature fluctuations reduce to the SachsWolfe fluctuations. The (total) cosmological dipole is thus suppressed with respect to the quadrupole (this remarkable property was stressed in [14]). If this case describes the reality we live in, the conclusion is that the observed dipole is necessarily of local origin.

For isocurvature perturbations, the intrinsic contribution cannot be neglected and the total window function $\mathcal{W}_{l}(k)$ is the sum of the SW window function and of the intrinsic window function which, using (13) and (18), as well as (19), is found to be

$$
\mathcal{W}_{l}^{i s o}(k)=5 j_{l}\left(k r_{l s}\right) \text {. }
$$

Hence $\mathcal{W}_{1}$ behaves like $k r_{l s}$ for small $k$ and can be made as large as one wishes with respect to the quadrupole window function by considering large enough scales.

The above analysis has been dealing with individual modes. To get the total multipoles, one must sum on all the modes. Assuming that $\Phi$ is a gaussian random field described by the power spectrum

$$
\left\langle\Phi_{k l m} \Phi_{k^{\prime} l^{\prime} m^{\prime}}\right\rangle=2 \pi^{2} k^{-3} \mathcal{P}_{\Phi}(k) \delta\left(k-k^{\prime}\right) \delta_{l l^{\prime}} \delta_{m m^{\prime}}
$$

the expectation value of the various multipoles reads

$$
\left\langle\left|a_{l m}\right|^{2}\right\rangle=\frac{4 \pi}{9} \int \frac{d k}{k} \mathcal{P}_{\Phi}(k) \mathcal{W}_{l}^{2}(k) .
$$

Once the power spectrum is given, the difference between the multipoles comes only from the window functions. The most common power spectrum is the flat spectrum corresponding to a constant $\mathcal{P}_{\Phi}(k)$. On Fig. 1, we have plotted the square of the dipole and quadrupole window functions in the adiabatic case. Since the dipole window function is always subdominant with respect to the quadrupole one or at best of the same order of magnitude for $k \sim h_{0}$, the conclusion is that the observed dipole can only be explained by a local effect in that case. On the contrary, for isocurvature perturbations, Fig.2 shows that the dipole window function dominates on ultra large scales and the observed dipole can be of cosmological origin. However the corresponding isocurvature spectrum cannot be constant on all scales because the main contribution to the dipole and quadrupole would come from scales $k \sim h_{0}$ and the resulting dipole and quadrupole would be 


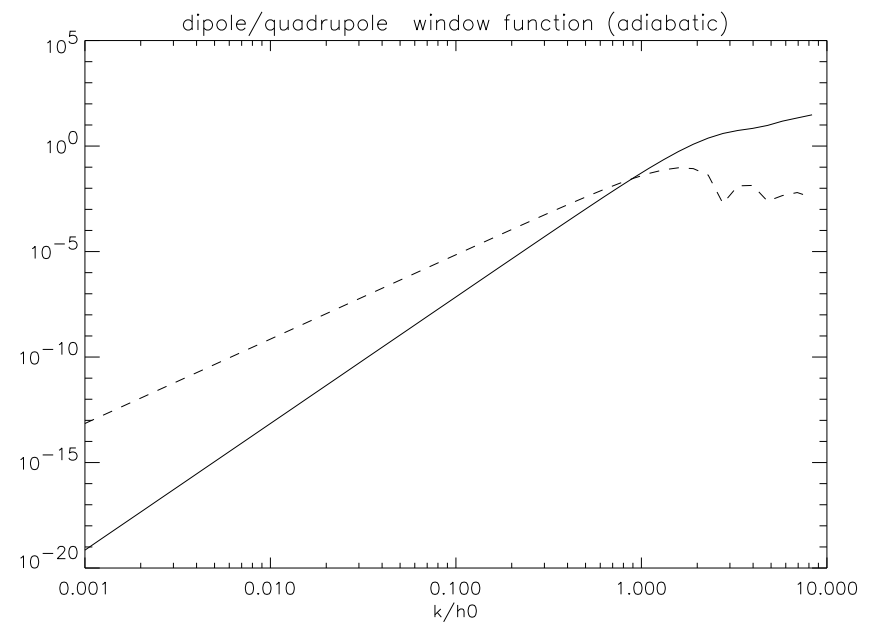

Figure 1: squared amplitude of the window functions in the adiabatic case for the dipole (solid curve) and the quadrupole (dashed curve). On ultra large scales the dipole window function is suppressed with respect to the quadrupole one.

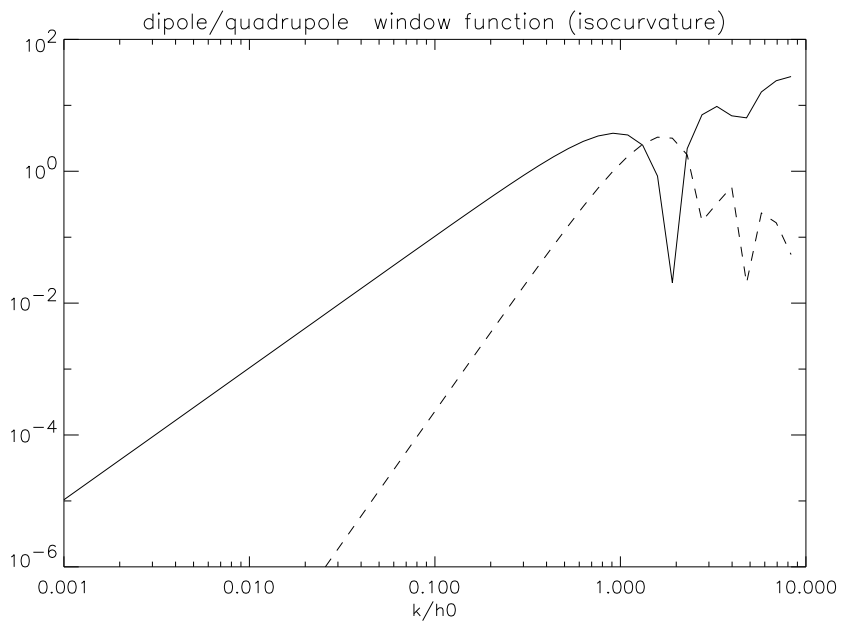

Figure 2: squared amplitude of the window functions in the isocurvature case for the dipole (solid curve) and the quadrupole (dashed curve). On ultra large scales the quadrupole window function is suppressed with respect to the dipole one. 
comparable. To obtain cosmological dipole and quadrupole compatible with the observations, two conditions must therefore be satisfied: the existence of ultra large scale isocurvature perturbations; and their spectrum must be suppressed for scales $\lambda<100 H_{0}^{-1}$. Although this cut-off condition can appear on first thought difficult to fulfill in a sensible model, it turns out that two relatively simple ideas can produce such an effect. One idea is the existence of pre-inflationnary isocurvature perturbations, which would have been pushed away far beyond the horizon during the inflation era [15]; the second idea is to consider the simplest two scalar field inflation model, which naturally produces an isocurvature spectrum with an abrupt cut-off [16].

\section{Open universe}

We now write the background spatial metric in the form

$$
d l^{2}=d r^{2}+\sinh ^{2} r\left(d \theta^{2}+\sin ^{2} \theta d \phi^{2}\right)
$$

With this choice of coordinates the Friedmann equation reads

$$
H^{2}=\frac{8 \pi G}{3} \rho+\frac{1}{a^{2}}
$$

where $H=a h$ is the Hubble parameter. As is clear from the above formula, $a$ represents the curvature scale: on scales smaller than $a$, the curvature of space is not "felt" whereas scales larger than $a$ are affected by the curvature. It is usual to parametrize the space curvature by the ratio of the energy density with respect to the critical energy density today, $\Omega_{0}=3 H_{0}^{2} / 8 \pi G$. $\Omega_{0}=1$ for a flat space and $\Omega_{0}<1$ for an open space. It then follows, in the matter era, that the comoving Hubble parameter can be expressed in terms of the redshift $z$ and $\Omega_{0}$ :

$$
h=\sqrt{\frac{1+\Omega_{0} z}{1-\Omega_{0}} .}
$$

In particular $h_{0}=1 / \sqrt{1-\Omega_{0}}$ represents the ratio between the current curvature scale $a_{0}$ and the Hubble scale $H_{0}^{-1}$. For an open universe the curvature scale is thus always larger than the Hubble scale and all the more larger as $\Omega_{0}$ is closer to one. 
In an open universe, the Fourier treatment does not apply. One must then use the formalism of mode functions for an hyperbolic space. Following [6], any gaussian random field can be expanded in the form

$$
f(r, \theta, \phi, \eta)=\int_{0}^{\infty} d k \sum_{l=0}^{\infty} \sum_{m=-l}^{l} f_{k l m}(\eta) Q_{k l m}
$$

where the $Q_{k l m}$ are eigenfunctions of the spatial Laplacian with eigenvalues $-k^{2}$. They can be chosen of the form

$$
Q_{k l m}=\Pi_{k l}(r) Y_{l m}(\theta, \phi),
$$

where $Y_{l m}(\theta, \phi)$ are the usual spherical harmonics on the two-sphere. The radial mode functions $\Pi_{k l}$ can be classified into two categories depending on the sign of $q^{2} \equiv k^{2}-1$. The radial functions for the subcurvature modes, corresponding to the values $q^{2}>0$, are given by

$$
\Pi_{k l} \equiv N_{k l} \tilde{\Pi}_{k l}
$$

with

$$
N_{k l}=\sqrt{\frac{2}{\pi}} q^{2}\left[\prod_{n=0}^{l}\left(n^{2}+q^{2}\right)\right]^{-1 / 2}, \quad \tilde{\Pi}_{k l}=q^{-2}(\sinh r)^{l}\left[\frac{-1}{\sinh r} \frac{d}{d r}\right]^{l+1} \cos (q r) .
$$

The subcurvature modes provide a complete orthonormal basis for square integrable functions and for this reason were the only modes taken into account by cosmologists. However, as stressed recently by Lyth and Woszczyna [6], they are not enough to describe properly gaussian random fields and one must also include the supercurvature modes, corresponding to the values $-1<q^{2}<0$. Their radial functions are obtained from the subcurvature modes by analytic continuation:

$$
\Pi_{k l} \equiv N_{k l} \tilde{\Pi}_{k l}
$$

with

$$
N_{k 0}=\sqrt{\frac{2}{\pi}}|q|, \quad N_{k l}=\sqrt{\frac{2}{\pi}}|q|\left[\prod_{n=1}^{l}\left(n^{2}+q^{2}\right)\right]^{-1 / 2} \quad(l>0)
$$


and

$$
\tilde{\Pi}_{k l}=|q|^{-2}(\sinh r)^{l}\left[\frac{-1}{\sinh r} \frac{d}{d r}\right]^{l+1} \cosh (|q| r) .
$$

The normalization of the mode functions is such that

$$
\int_{0}^{\infty} \Pi_{k l}(r) \Pi_{k^{\prime} l^{\prime}}(r) \sinh ^{2} r d r=\delta\left(q-q^{\prime}\right) \delta_{l l^{\prime}}
$$

Moreover for a homogeneous gaussian random field, one defines the power spectrum by

$$
\left\langle f_{k l m} f_{k^{\prime} l^{\prime} m^{\prime}}^{*}\right\rangle=\frac{2 \pi^{2}}{k|q|^{2}} \mathcal{P}_{f}(k) \delta\left(k-k^{\prime}\right) \delta_{l l^{\prime}} \delta_{m m^{\prime}}
$$

Note that in the flat space limit, corresponding to the limits $k \rightarrow \infty$ and $r \rightarrow 0$ with $k r$ fixed,

$$
\Pi_{k l}(r) \rightarrow \sqrt{\frac{2}{\pi}} k j_{l}(k r),
$$

and one recovers equation (33) for the power spectrum.

In contrast with the flat case, the gravitational potential modes $\Phi_{k l m}$ on scales larger than the Hubble radius evolve with time. Their evolution is given by the linearized Einstein equations (see e.g. [12]):

$$
\Phi_{k}(\eta)=F(\eta) \tilde{\Phi}_{k}
$$

with

$$
F(\eta)=5 \frac{\sinh ^{2} \eta-3 \eta \sinh \eta+4 \cosh \eta-4}{(\cosh \eta-1)^{3}} .
$$

(with the normalization $a=\cosh \eta-1$ for the scale factor). $\mathrm{F}$ is normalized so that $F \rightarrow 1$ when $\eta \rightarrow 0$. $\tilde{\Phi}_{k}$ is constant in time and is given by the initial spectrum at the beginning of the matter era. At that initial stage, the curvature can be ignored. In fact, even for small values of $\Omega_{0}, F(\eta)$ remains almost constant during most of the matter era and drops suddenly in the recent past. Similarly, the velocity potential obeys to a law different from the flat case and given by

$$
h V(\eta)=-\frac{2}{3} G(\eta) \tilde{\Phi}
$$


with

$$
G(\eta)=\frac{15(2 \eta+\eta \cosh \eta-3 \sinh \eta) \sinh \eta}{16 \sinh ^{6}(\eta / 2)} .
$$

Like $F, G$ has been normalized such that $G \rightarrow 1$ when $\eta \rightarrow 0$ and $G$ is significantly different from one only for small redshifts.

The decomposition of the Sachs-Wolfe terms in (11) on the basis $Q_{k l m}$, like in (38), yields for the harmonic coefficients the expression

$$
a_{l m}=\sqrt{\frac{2}{\pi}} \int_{0}^{\infty} d k|q| \frac{1}{3} \tilde{\Phi}_{k l m}\left[\mathcal{W}_{k l}^{p S W}+\mathcal{W}_{k l}^{D o p}+\mathcal{W}_{k l}^{I S W}\right],
$$

with

$$
\begin{gathered}
\mathcal{W}_{k l}^{p S W}=\left(3 F_{l s}-2 G_{l s}\right) \hat{\Pi}_{k l}\left(r_{l s}\right) /|q|, \\
\mathcal{W}_{k l}^{D o p}=2 h_{l s}^{-1} G_{l s} \partial_{r} \hat{\Pi}_{k l}\left(r_{\mathrm{ls}}\right) /|q|-\frac{2}{3} h_{0}^{-1} G_{0} k \delta_{l, 1},
\end{gathered}
$$

and

$$
\mathcal{W}_{k l}^{I S W}=\left[-6 F_{l s} \hat{\Pi}_{k l}\left(r_{\mathrm{ls}}\right)+6 \int_{0}^{r_{\mathrm{ls}}} d r F\left(\eta_{\mathrm{ls}}+r_{\mathrm{ls}}-r\right) \partial_{r} \hat{\Pi}_{k l}(r)\right] /|q| .
$$

Except for the flat case limit, we shall take $F_{l s}=G_{l s}=1$ in the above expressions, which is an excellent approximation because $z_{l s}>>1$. A hat means a division by $\sqrt{2 / \pi}$. $h_{\mathrm{ls}}$ is the comoving Hubble parameter at the time of last scattering. $r_{\mathrm{ls}}$ is the radius coordinate corresponding to the emission of the photons, i.e. the coordinate of the last scattering surface. This radius can be related to the redshift $z_{\mathrm{ls}}$ via the relation

$$
\sinh r(z)=\frac{2}{h_{0}}(1+z)^{-1} \Omega_{0}^{-2}\left[\Omega_{0} z+\left(\Omega_{0}-2\right)\left(\left(1+\Omega_{0} z\right)^{1 / 2}-1\right)\right] .
$$

We must also consider the intrinsic contribution $a_{l m}^{\text {int }}$ to the harmonic coefficients. For adiabatic fluctuations, like in the flat case, the intrinsic contribution is negligible as can be seen from (16): the reason is not this time the smallness of $k^{2}$ but the fact that $h^{2}>>1$ (see (37)). For isocurvature perturbations, the intrinsic window function is given by

$$
\mathcal{W}_{k l}^{i s o}=5 F_{l s} \hat{\Pi}_{k l}\left(r_{\mathrm{ls}}\right) /|q|
$$


Finally, once a power spectrum is given, the expectation values for the harmonic coefficients can then be written in the form

$$
\left\langle\left|a_{l m}\right|^{2}\right\rangle=\frac{4 \pi}{9} \int \frac{d k}{k} \mathcal{P}_{\Phi}(k) \mathcal{W}_{l}^{2}(k),
$$

where $\mathcal{W}_{l}(k)$ is the sum of all the relevant window functions.

\subsection{Ultra large scale limit}

In the flat case, it was possible to obtain a dipole much larger than the quadrupole with isocurvature perturbations on scales much larger than the Hubble radius. We wish now to consider such scales in an open universe. In the ultra large scale limit, corresponding to $k \rightarrow 0$, the general expressions can be simplified because $\Pi_{k l}(r) \sim k f_{l}(r)$. Following [7], we define

$$
N_{l} \equiv \lim _{k \rightarrow 0} k N_{k l}=\sqrt{\frac{2}{\pi}} \prod_{n=2}^{l}\left(n^{2}-1\right)^{-1 / 2}
$$

for $l>1$ and $N_{1}=\sqrt{2 / \pi}$. Similarly, we define

$$
\tilde{\Pi}_{l} \equiv \lim _{k \rightarrow 0} \tilde{\Pi}_{k l} / k^{2}
$$

The expressions we are mainly interested in are

$$
\tilde{\Pi}_{1}(r)=\frac{1}{2}\left(\operatorname{coth} r-\frac{r}{\sinh ^{2} r}\right)
$$

and

$$
\tilde{\Pi}_{2}(r)=\frac{1}{2}\left[1+3 \frac{1-r \operatorname{coth} r}{\sinh ^{2} r}\right] .
$$

The window functions (53 55) can then be expressed in the limit $k \rightarrow 0$ in the form

$$
\mathcal{W}_{k l} \sim k \mathcal{L}_{l}\left(\Omega_{0}, z_{\mathrm{ls}}\right)
$$

with

$$
\mathcal{L}_{l}^{p S W}=\hat{N}_{l} \tilde{\Pi}_{l}\left(r_{\mathrm{ls}}\right), \quad \mathcal{L}_{l}^{D o p}=2 \hat{N}_{l} h_{\mathrm{ls}}^{-1} \frac{d}{d r} \tilde{\Pi}_{l}\left(r_{\mathrm{ls}}\right)-\frac{2}{3} h_{0}^{-1} G_{0} \delta_{l, 1},
$$




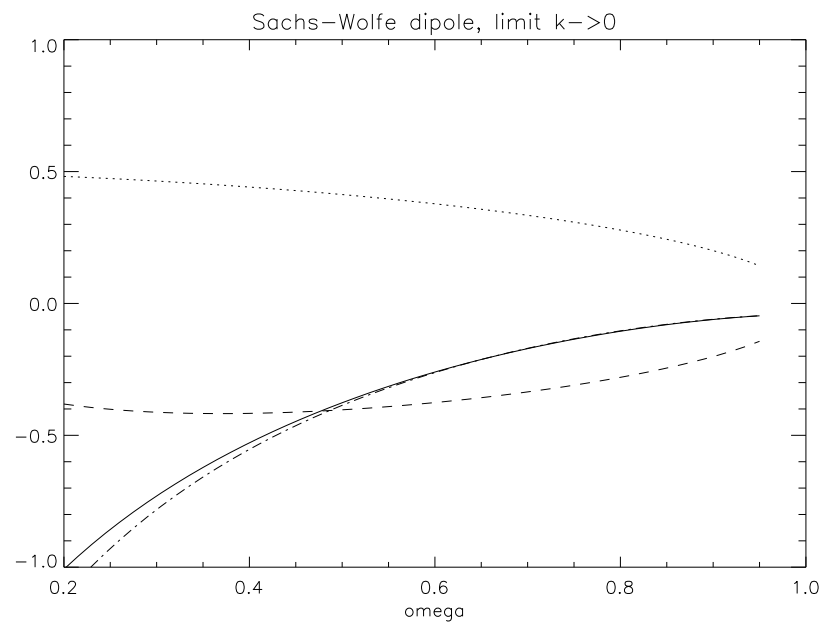

Figure 3: amplitude of the Sachs Wolfe dipole window functions in the limit $k \rightarrow 0$ as a function of $\Omega_{0}$. The total Sachs-Wolfe window function (solid curve) is the sum of the pure Sachs-Wolfe contribution (dotted curve), of the Doppler contribution (dashed curve), which almost cancel each other, and finally of the ISW contribution (dashed-dotted curve). 


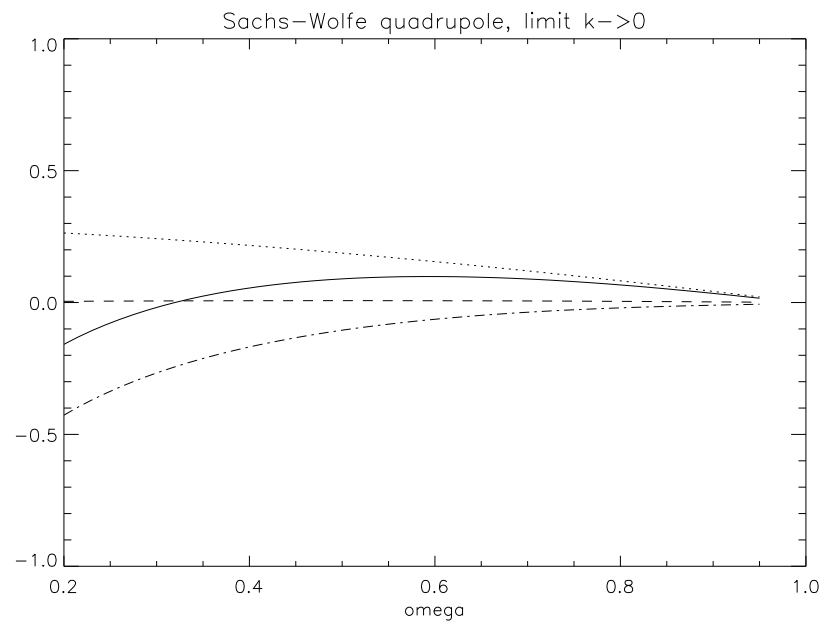

Figure 4: amplitude of the Sachs Wolfe quadrupole window functions in the limit $k \rightarrow 0$ as a function of $\Omega_{0}$. In the total Sachs-Wolfe window function (solid curve), The Doppler term (dashed curve) is very small with respect to the two other terms, the pure SW term (dotted curve) and the ISW term (dashed-dotted curve). One notices that the SW dipole vanishes near $\Omega_{0} \simeq 0.3$. 
and

$$
\mathcal{L}_{l}^{I S W}=\hat{N}_{l}\left[-6 \tilde{\Pi}_{l}\left(r_{\mathrm{ls}}\right)+6 \int_{0}^{r_{\mathrm{ls}}} d r F\left(\eta_{\mathrm{ls}}+r_{\mathrm{ls}}-r\right) \frac{d}{d r} \tilde{\Pi}_{l}(r)\right] .
$$

These three quantities, as well as their sum corresponding to the total SachsWolfe effect are plotted as functions of $\Omega_{0}$ for the dipole (Fig. 3) and for the quadrupole (Fig. 4). The first striking difference with the flat case is that the Sachs-Wolfe dipole is not suppressed with respect to the quadrupole. One can see more precisely on Fig. 3 that this is due principally to the ISW contribution, even if the pure SW and Doppler contributions do not cancel each other exactly, especially for low $\Omega_{0}$. As for the quadrupole, one can see that the Doppler contribution is extremely small in comparison with the other terms. Another remark is that the quadrupole vanishes around $\Omega_{0} \simeq 0.3$ for which the pure SW effect and the ISW effect happen to compensate each other.

In Fig. 5 and Fig. 6, we have plotted, still in the ultra large scale limit, the total window functions for the multipoles $l=1,2,3$ with, respectively, adiabatic and isocurvature initial conditions. We have added the multipole $l=3$ to show that even if the dipole can be made much bigger than the quadrupole in the zone $\Omega_{0} \simeq 0.3$, this cannot explain the observed CMBR since the next multipole is roughly one tenth of the dipole. The conclusion is therefore that, in an open universe, the cosmological dipole is typically of the same order of magnitude as the higher multipoles. Within our assumptions, the observed dipole can be explained cosmologically only in a flat space. In the next section, we examine in detail this flat space limit in order to put a limit on the flatness required to explain the dipole by cosmological perturbations.

\subsection{Flat space limit}

We saw in the previous subsection that, for supercurvature modes, the window functions go to zero when $\Omega \rightarrow 1$. We need to examine now in more details what is the relative importance of the dipole with respect to the quadrupole in this limit. To do so, it is convenient to introduce the paramater

$$
\epsilon=\sqrt{1-\Omega_{0}}
$$




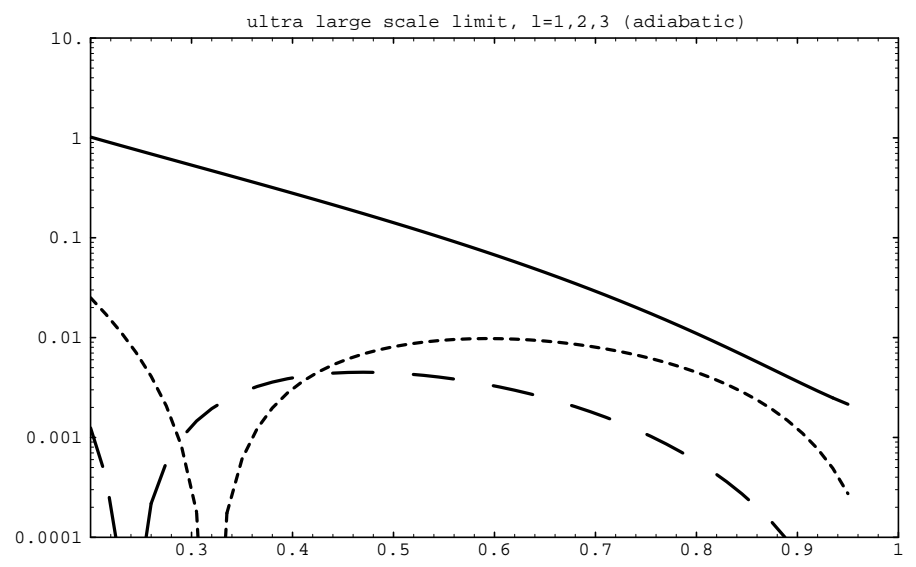

Figure 5: squared amplitude of the total window functions (intrinsic and Sachs-Wolfe) for ultra large scale adiabatic perturbations (limit $k \rightarrow 0$ ), for the three multipoles $l=1,2,3$ (from top to bottom). 


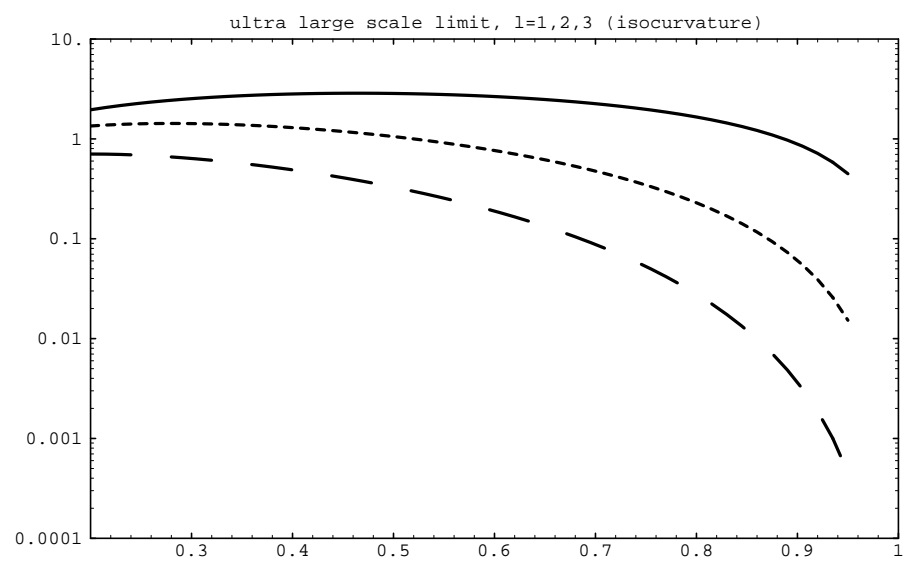

Figure 6: squared amplitude of the total window functions (intrinsic and Sachs-Wolfe) for ultra large scale isocurvature perturbations (limit $k \rightarrow 0$ ), for the three multipoles $l=1,2,3$ (from top to bottom). 
One can immediately notice that the curvature radius reads $a_{0}=H_{0}^{-1} / \epsilon$ so that the curvature radius goes to infinity with respect to the Hubble radius, as one can expect in a flat space limit. Expanding (56) in $\epsilon$, one finds

$$
\begin{aligned}
r\left(\Omega_{0}, z\right)= & 2\left(1-\frac{1}{\sqrt{1+z}}\right) \epsilon+\left[\frac{2}{1+z}\left(z+3-3 \sqrt{1+z}+\frac{z}{2 \sqrt{1+z}}\right)\right. \\
& \left.-\frac{4}{3}\left(1-\frac{1}{\sqrt{1+z}}\right)^{3}\right] \epsilon^{3}+\mathcal{O}\left(\epsilon^{5}\right) .
\end{aligned}
$$

The expansion of the terms appearing in the total Sachs-Wolfe effect is

$$
\begin{aligned}
\mathcal{W}_{k 1}^{p S W}= & \frac{2}{3} k\left(1-\frac{1}{\sqrt{1+z}}\right) \epsilon+\frac{2}{3} k\left[\left(z+\frac{11}{7}-\frac{22+35 z}{14 \sqrt{1+z}}\right) /(1+z)\right. \\
& \left.-\frac{2}{5}\left(3+k^{2}\right)\left(1-\frac{1}{\sqrt{1+z}}\right)^{3}\right] \epsilon^{3}+\mathcal{O}\left(\epsilon^{5}\right), \\
\mathcal{W}_{k 1}^{D o p}= & -\frac{2}{3} k\left(1-\frac{1}{\sqrt{1+z}}\right) \epsilon+\frac{2}{3} k\left[\frac{1}{7}+\frac{1}{\sqrt{1+z}}\left(\frac{7 z-2}{14(1+z)}\right.\right. \\
& \left.\left.-\frac{2}{5}\left(4+3 k^{2}\right)\left(1-\frac{1}{\sqrt{1+z}}\right)^{2}\right)\right] \epsilon^{3}+\mathcal{O}\left(\epsilon^{5}\right), \\
\mathcal{W}_{k 1}^{I S W}= & -\frac{16 k}{21}\left(1-\frac{1}{\sqrt{1+z}}\right)^{2}\left(1+\frac{2}{\sqrt{1+z}}\right) \epsilon^{3}+\mathcal{O}\left(\epsilon^{5}\right) .
\end{aligned}
$$

Note that the strict limit $\Omega_{0}=1$ is not obtained simply by setting $\epsilon=0$, which would give zero for all the terms above. Indeed it makes more sense to label the perturbation scales by the physical quantity $k / h_{0}$ instead of the unphysical wavenumber $k$. Since $h_{0}=1 / \epsilon$, the proper flat limit is given by $\epsilon \rightarrow 0$ with $k \epsilon$ fixed. However, this limit applies only to subcurvature modes whereas supercurvature modes correspond to larger and larger scales as $\Omega_{0} \rightarrow 1$ since they are bounded by $k<1$. The most striking feature of (68-70) is that the dominant terms of the pure SW contribution and of the Doppler term just cancel each other. It is thus clear that the suppression of the dipole is really a consequence of the flatness. We have also written 
explicitly the terms at the order $\epsilon^{3}$ because they represent the leading order in the total SW window function. For the quadrupole, one obtains

$$
\begin{gathered}
\mathcal{W}_{k 2}^{p S W}=\frac{4}{15} k\left(k^{2}+3\right)^{1 / 2}\left(1-\frac{1}{\sqrt{1+z}}\right)^{2} \epsilon^{2}+\mathcal{O}\left(\epsilon^{4}\right), \\
\mathcal{W}_{k 2}^{D o p}=\frac{8}{15} k\left(k^{2}+3\right)^{1 / 2}\left(\frac{1}{\sqrt{1+z}}-\frac{1}{1+z}\right) \epsilon^{2}+\mathcal{O}\left(\epsilon^{4}\right), \\
\mathcal{W}_{k 1}^{I S W}=\mathcal{O}\left(\epsilon^{4}\right) .
\end{gathered}
$$

For isocurvature, one must add the intrinsic contribution which is roughly

five times $\mathcal{W}_{k l}^{p S W}$. By comparing (68,70) with (71-73), one can see that for $k<<1$ all the window functions, both for the dipole and the quadrupole, are proportional to $k$. As a consequence one cannot obtain a significant dipole by considering sufficiently large scales, like in the flat case. The only possible difference between the dipole and the quadrupole can thus come only from the parameter $\epsilon$. For adiabatic perturbations, the cancellation of the $\epsilon$ terms implies that the dipole is $\epsilon$ times the quadrupole. On the contrary, for isocurvature perturbations, the total dipole is proportional to $\epsilon$ and can be much bigger than the quadrupole which goes like $\epsilon^{2}$. To obtain a dipole $10^{2}$ times larger than the quadrupole, one thus needs $\epsilon<10^{-2}$, i.e. $\left|\Omega_{0}-1\right|<10^{-4}$.

\section{Conclusions}

The main conclusion of this paper is that a cosmological origin for the dipole (or a significant part of it) must be rejected for an open cosmology, which means that the observed dipole would be in that case essentially a Doppler effect resulting from our local motion dominated by small scale density perturbations. This conclusion assumes that the universe can be described as a FLRW model with gaussian fields of linear perturbations. More exotic possibilities have not been considered here.

Another conclusion of this work is that the suppression in a flat universe of the dipole due to ultra large scale adiabatic perturbations, with respect to the quadrupole, is no longer true in an open universe. 
It turns out that the only possibility to get a dipole two orders of magnitude bigger than the other multipoles is a model with isocurvature perturbations on scales of the order of $10^{2} \mathrm{H}_{0}^{-1}$ in an almost flat geometry: the constraint is $\left|\Omega_{0}-1\right|<10^{-4}$. This constraint can also be interpreted as demanding that the curvature scale be one hundred timed larger than the Hubble radius. When this constraint is satisfied but with $\Omega_{0}$ non strictly equal to one (this would be the case for example of an open universe after a phase of inflation), two kinds of perturbations can produce the observed dipole : the subcurvature modes on scales larger than one hundred times the Hubble radius, like in the flat case; but also the supercurvature modes, which do not exist in the flat case.

To conclude, a reliable measurement of $\Omega_{0}$ (see [17] for a discussion on measuring $\Omega_{0}$ ) different from one would strongly confirm the Doppler assumption for the dipole. Conversely, if unquestionable cosmological observations conclude that our local motion is in disagreement with the CMBR dipole, then it would suggest that we live in a flat universe and that ultra large scale isocurvature perturbations must exist.

\section{Acknowledgement.}

I would like to thank N. Deruelle, T. Piran and D. Wands for stimulating discussions.

\section{References}

[1] A. Kogut et al., Astrophys. J. 419, 1 (1993)

[2] Lauer, T.R. and M. Postman, Astrophys. J., 425, 418 (1991)

[3] B. Paczynski, T. Piran, Astrophys. J. 364, 341 (1990)

[4] D. Langlois, T. Piran, Phys. Rev. D53, 2908 (1996)

[5] L.P. Grishchuk, Y.B. Zeldovich, Astron. Zh. 55, 209 (1978) [Sov. Astron. 22,125 (1978)]

[6] D.H. Lyth, A. Woszczyna, Phys. Rev. D52, 3338 (1995) 
[7] J. Garcia-Bellido, A.D. Liddle, D.H. Lyth and D. Wands, Phys. Rev. D52, 6750 (1995)

[8] Panek, Phys. Rev. D 34, 416 (1986)

[9] E.W. Kolb and M.S. Turner, The early Universe, Addison Wesley 1990

[10] J.M. Bardeen, Phys. Rev. D 22, 1882 (1980)

[11] R.K. Sachs, A.M. Wolfe, Ap. J. 147, 73 (1967).

[12] V.F. Mukhanov, H.A. Feldman, R.H. Brandenberger, Phys. Reports 215, $203(1992)$

[13] H. Kodama, M. Sasaki, Int. J. Mod. Phys. A1, 265 (1986)

[14] M. Bruni, D.H. Lyth, Phys. Lett. B 323, 118 (1994)

[15] M. S. Turner, Phys. Rev. D 44, 3737 (1991)

[16] D. Langlois, Phys. Rev. D 54, 2447 (1996)

[17] A. Dekel, D. Burstein, S.D.M. White, "Measuring Omega", astro$\mathrm{ph} / 9611108$ 